Revista Brasil. Bot., V.31, n.3, p.537-548, jul.-set. 2008

\title{
Morfologia de esporos de espécies de Polytrichaceae Schwägr. (Bryophyta) do Brasil ${ }^{1}$
}

\author{
LUCAS MATHEUS DA ROCHA ${ }^{2,4}$, VANIA GONÇALVES-ESTEVES ${ }^{2}$ e \\ ANDRÉA PEREIRA LUIZI-PONZO 3
}

(recebido: 22 de junho de 2006; aceito 10 de agosto de 2008)

\begin{abstract}
Spore morphology of Polytrichaceae Schwägr. (Bryophyta) from Brazil). Spore morphology is described for all six genera of Brazilian Polytrichaceae based on light and scanning electron microscopy study of 13 species of the approximately 30 species of the family native to the country. Discussion on its taxonomic importance is also provided. Spores were analyzed before and after the acetolysis method. Polytrichaceae is eurypalynous. The spore surface of Atrichum androgynum (Müll. Hal.) A. Jaeger has regular granules and isolated gemmae. The spores of Itatiella P. Beauv. and Oligotrichum Lam. \& DC. species are small to medium size, with bacula, elongated processes, clavae and pila on the surface. Species of Pogonatum P. Beauv. gathers spores of very small to small size, granulate surface. Polytrichadelphus pseudopolytrichum (Raddi) G. L. Sm. spores are very small to small size, and ornamentation formed by reduced smooth spines. In Polytrichum Hedw. species the spores are small and ornamentation is formed by ornamented spine-like processes to granules.
\end{abstract}

Key words - bryophytes, mosses, palynology, Polytrichaceae

RESUMO - (Morfologia de esporos de espécies de Polytrichaceae Schwägr. (Bryophyta) do Brasil). A morfologia dos esporos é descrita para todos os seis gêneros de Polytrichaceae ocorrentes no Brasil baseando-se no estudo, em microscopia de luz e eletrônica de varredura, de 13 das aproximadamente 30 espécies da família nativas para o país. Também é apresentada a discussão sobre sua importância taxonômica. Os esporos foram estudados antes e após o método da acetólise. Os resultados indicam Polytrichaceae como euripalinológica. A superfície dos esporos de Atrichum androgynum (Müll. Hal.) A. Jaeger é formada por grânulos regulares, com gemas isoladas. Os esporos de Itatiella ulei P. Beauv. e das espécies de Oligotrichum Lam. \& DC. são de tamanho pequeno a médio, com báculos, processos alongados, clavas e pilos na superfície. Espécies de Pogonatum P. Beauv. possuem esporos de tamanho muito pequeno a pequeno, com superfície granulada. Os esporos de Polytrichadelphus pseudopolytrichum (Raddi) G. L. Sm. são de tamanho muito pequeno a pequeno, com ornamentação formada por diminutos espinhos lisos. Nas espécies de Polytrichum Hedw., os esporos são de tamanho pequeno e a ornamentação é formada por processos espinhosos ornamentados a grânulos.

Palavras-chave - briófitas, musgos, palinologia, Polytrichaceae

\section{Introdução}

Polytrichaceae se caracteriza por possuir plantas ortotrópicas robustas a muito pequenas. Gametófitos variam de muito folhosos a protonemas persistentes; os filídios são papilosos ou lisos, com base curvada, alva e invaginante. A costa é forte, percurrente a excurrente. A lamela fotossintetizante está presente na superfície adaxial e, ocasionalmente, na superfície abaxial, ocupando parcial ou totalmente a lâmina. As cápsulas são eretas, angulares, variando de ovóides a cilíndricas, bilaterais a dorsiventrais. O peristômio é simples formado por 32

1. Parte da dissertação de Mestrado do primeiro autor, Programa de Pós-Graduação em Ciências Biológicas (Botânica) do Museu Nacional/Universidade Federal do Rio de Janeiro, Rio de Janeiro.

2. Universidade Federal do Rio de Janeiro, Museu Nacional, Laboratório de Palinologia, 25460-036 Rio de Janeiro, RJ, Brasil.

3. Universidade Federal de Juiz de Fora, Departamento de Botânica, 36036-900 Juiz de Fora, MG, Brasil.

4._Autor para correspondência: lucaskogima@yahoo.com.br a 64 (raramente 16) dentes, unidos na extremidade pelo epifragma. A caliptra é cuculada, extremamente pilosa ou, muito raramente, glabra (Smith 1971, Crum 1976, Schofield 1985, Hyvönen 1989, Buck \& Goffinet 2000).

No Brasil, as Polytrichaceae são representadas por seis gêneros e 30 espécies (Yano 1996). No presente trabalho, foram estudados os esporos de 13 espécies que representam os seis gêneros que ocorrem no Brasil.

O estudo palinológico de diferentes espécies, tanto de Fanerógamas (Bove 2000, Bove \& Melhem 2000, Gonçalves-Esteves \& Melhem 2000), quanto de Briófitas (Mogensen 1981, 1983, Luizi-Ponzo et al. 1997, LuiziPonzo \& Barth 1998, 1999, Luizi-Ponzo \& Melhem 2006) e Pteridófitas (Ferrarini et al. 1986, Esteves \& Melhem 1992, Sylvestre 1995), tem demonstrado possuir grande importância na circunscrição de grupos taxonômicos e possibilitado a aquisição de um maior conhecimento da morfologia desses grupos.

No que diz respeito aos estudos palinológicos que analisaram espécies de Polytrichaceae destacam-se 
Erdtman $(1957,1965)$ que caracterizou os esporos de quatro gêneros e dez espécies da família, Smith (1974), que utilizou dados palinológicos e a estrutura do epifragma para reorganizar as delimitações entre gêneros, e Stefureac $e t$ al. (1979), que examinaram a ornamentação da superfície de oito espécies sob microscopia eletrônica de varredura.

Alguns estudos abordaram esporos de um pequeno número de espécies da família, como Mullen \& Frye (1947), McClymont \& Larson (1964), Nyholm (1971), Smith (1971) e Menzel (1985). Pode-se destacar o trabalho de Farias (1984) que descreveu, sob microscopia eletrônica de varredura, os esporos de algumas espécies de Polytrichaceae ocorrentes no Brasil.

Hyvönen (1989) considerou o esporo e sua ultraestrutura caracteres importantes a serem considerados em Polytrichaceae. Segundo o autor, esporos pequenos com projeções cônicas na superfície representariam um estado de caráter apomórfico para a família.

\section{Material e métodos}

Foram estudados os esporos de 13 espécies pertencentes a seis gêneros da família Polytrichaceae Schwägr. ocorrentes no Brasil, provenientes de diferentes herbários brasileiros, a saber BHCB, CESJ, MG, HB, PACA, R, RB e SP, cujos acrônimos estão de acordo com Holmgren et al. (1990).

Material estudado: Atrichum androgynum (Müll. Hal.) Jaeger: BRASIL. Rio Grande do Sul: Dois Irmãos, 23-X-1971, A. Sehnem 83004 (PACA); São Francisco de Paula, 15-VIII-1965, A. Sehnem 83016 (PACA)*. Itatiella ulei (Broth. ex Müll. Hal.) G.L. Sm.: BRASIL. Rio DE JANEIRO: Serra de Itatiaia, 19-III-1987, O. Yano s.n. (RB210623)*. Espírito Santo: Parque Nacional do Caparaó, 16-IX-1984. D.M. Vital \& W.R. Buck 11764 (RB). Minas Gerais: Parque Nacional de Itatiaia, 27-VI-1974, D.M. Vital 3631 (SP); Itamonte, 30-X-1994, O. Yano \& Z.R. Melo 22539 (SP). Oligotrichum denudatum Merril: BRASIL. Minas GeRAIs: São Roques de Minas, 19-IX-1984, Vital \& Buck V 11941 (SP)*. Oligotrichum riedelianum (Mont.) Mitt.: BRASIL. Minas Gerais: Itamonte, 1-V-1994, O. Yano \& Z.R. Melo 22628 (SP)*. RIO DE JANEIRO: Reserva Florestal de Itatiaia, 4-IX-1924, Pedro Occhioni s.n. (RB218309); Itatiaia, 6-IX-1955, Fidalgo \& Kauffman Fidalgo Eg - 7 (RB); Serra do Itatiaia, 6-VI-1902, P. Dusén 493 (R). Rio Grande do Sul: São Francisco de Paula, 18-XII-1950, A. Sehnem 83017 (PACA). Pogonatum brasiliense (Hampe) A. Jaeger: BRASIL. Rio DE JANEIRo: Itatiaia, 20-X-1999, J.M.A. Braga 5252 et al. (RB)*. Pogonatum campylocarpum (Müll. Hal.) Mitt.: BRASIL. MinAS GERAIS: Itamonte, 21-IV-1995, O. Yano et al. 23861 (SP). São Paulo: Bocaina, 9-IV-1896, Löfgren \& Edwall 537 (SP)*. Rio DE JANeIRo: Itatiaia, 18-I-1925, Brotherus s.n. (RB218294); Nova Friburgo, 8-VI-1977, G. Martinelli 2460 et al. (RB). Pogonatum pensilvanicum (Hedw.) P. Beauv.: BRASIL. MinAs GeRAIs: Cipó, 14-III-1967, D.M.
Vital 1236 (SP); Itumirim, 10-IV-1980, D.M. Vital 8856 (SP); Parque Estadual do Ibitipoca, 4-VII-1975, L. Krieger 13949 (CESJ)*; Ouro Preto, 9-II-1976, D.M. Vital 4427 (SP); Santa Bárbara, 27-V-1983, O. Yano \& J.R. Pirani 7298 (SP). Pogonatum tortile (Sw.) Brid.: BRASIL. Minas GERAIS: Ouro Preto, II-1892, E. Ule 1319 (R). Rio DE JANEIRo: Itatiaia, 16-I-1925, Brotherus s.n. (RB218302)*. Polytrichadelphus pseudopolytrichum (Raddi) G.L. Sm.: BRASIL. MINAS GERAIs: Itamonte, 21-IV-1995, O. Yano 23875 et al. (SP); Serra do Picú, 10-XII-1886, L. Nette s.n. (R14259); Caraça, III-1892, E. Ule 1313 (R); idem, V-1980, T. Vianna s.n. (BHCB2005); Serra de Ouro Preto, II-1892, E. Ule 1314 (R)*; idem, s.d., E. Ule s.n. (R82777). Rio DE JANeIro: Itatiaia, X-1957, Hermendorff 10580 (R). Rio Grande do Sul: Aparados, 14-I-1942, A. Sehnem s.n. (PACA83028). Polytrichum commune L ex. Hedw.: BRASIL. Minas Gerais: Serra do Picú, X-1957, Rangel et Schwacke s.n. (R14257)*, Serra de Ouro Preto, II-1892, E. Ule 1322 (R). Santa Catarina: São Bento, 28-VI-1957, Schwacke s.n. (R14260). PARANÁ: Jaguariaíva, 19-XII-1974, Kummrow 826 (PACA), Antonina, 24-III-1966, G. Hatschbach 14136 (PACA). ALEMANHA. Neuruppin, VI-1886, Warmstorf s.n. (R82772). Polytrichum juniperinum Hedw. var. juniperinum: BRASIL. MINAS GeraIs: Caeté, 6-V-1934, M. Barreto 10217 (BHCB); idem, V-1988, S. Eliasaro 672 (BHCB); idem, 18-IV-1981, T.S.M. Grandi \& L. Fiedler 630 (BHCB); Nova Lima, 17-VIII-1983, P.M. Andrade s.n. (BHCB); Passa Quatro, XI-1948, J. Vidal s.n. (R87701); Serra da Mantiqueira, I-1885, s. col. (R81554), idem, 1949, J. Vidal s.n. (R82740), idem, 1949, J. Vidal s.n. (R87685); Poços de Caldas, 17-III-1983. O. Yano \& D.P. Santos 6142 (SP); idem, 27-VII-1965, C. Emmerich 117 (HB); Caeté, 17-XI-1986, O. Yano \& P.I.S. Braga 10491 (SP)*; Caraça, III-1892, E. Ule 1316 (R); idem, 25-III-1957, E. Pereira 2638 \& Pabst 3474 (HB); Ouro Preto, II-1892, E. Ule 1321 (R); Itabira do Campo, IV-1892, E. Ule 1323 (R); Gandarela, 17-VII-1972, Luiz E.M.F. 3437 (R). Rio DE JANEIRO: Serra do Itatiaia, 13-V-1902, P. Dusén 192 (R). Polytrichum juniperinum var. paulense (Geh. \& Hampe) Herzog.: BRASIL. SANTA Catarina: Minas, II-1890, E. Ule s.n. (MG20122)*. Paraná: Quatro Barras, 12-IX-1967, A. Sehnem 9998 (PACA). Polytrichum subremotifolium Geh. \& Hampe.: BRASIL. PARANÁ: Palmeira, 21-VII-1968, G. Hatschbach 19527 (PACA)*.

Sob microscopia de luz (ML), para a observação de possíveis anomalias, todo o material botânico examinado teve os esporos preparados de acordo com Wodehouse (1935) e Erdtman (1960), empregando-se as alterações propostas por Luizi-Ponzo \& Melhem (2006).

Deste modo, as médias de tamanho dos diâmetros maiores (em vista polar) foram baseadas em 100 esporos, relacionados ao acaso em cinco lâminas, para o material padrão e 30 esporos, tomados ao acaso em três lâminas, do material de comparação. Foram calculadas a média $(\bar{x})$, o desvio padrão $(s)$, o erro padrão $\left(s_{x}\right)$ e as faixas de variação $\left(X_{\min }-X_{\text {maxx }}\right)$ de tamanho para todas as espécies. As medidas dos estratos do esporoderma foram realizadas em 10 esporos, em vista equatorial, em lâminas de Wodehouse (1935) e acetolisadas. 
Para observação sob microscopia eletrônica de varredura (MEV), os esporos foram dispersos sobre os suportes cobertos com fita dupla face, cobertos com camada de ouro de 20 nm e observados em um microscópio JEOL JSM 5800.

Os esporos foram descritos de acordo com o padrão e a terminologia adotados nos trabalhos publicados por Barth \& Melhem (1988) e Punt et al. (2007).

\section{Resultados}

Os táxons estudados da família Polytrichaceae apresentam esporos em mônades, de tamanho muito pequeno a médio com simetria radial, heteropolares, âmbito subcircular; forma plano-convexa ou côncavoconvexa, região apertural proximal, esporoderma formado por intina, exina e perina. A intina apresenta espessamento proximal (região apertural) e a exina diminuição de espessura na mesma região. Exina psilada, ornamentação formada pelos elementos da perina incluindo grânulos, báculos, pilos ou processos espinhosos ornamentados.
A ornamentação do esporoderma pode ser diferenciada entre o pólo proximal e o distal, caracterizando a heteropolaridade dos esporos, que está também relacionada às diferenças na espessura dos estratos do esporoderma. Na maioria dos esporos estudados, a polaridade pode ser facilmente observada.

Atrichum P. Beauv.

Atrichum androgynum (Müll. Hal.) A. Jaeger

Esporos isomórficos, de tamanho pequeno (tabela 1), heteropolares, âmbito subcircular, com região apertural proximal; superfície granulada (figuras 1-3).

A região apertural apresenta-se na forma subtriangular (figura 3) ou irregular e foi identificada em ML. São facilmente definidas as vistas polar e equatorial dos esporos (figura 1).

Os elementos de perina consistem de grânulos regulares na forma e no tamanho quando observados em ML, porém, em MEV foi constatada a presença de gemas isoladas e, eventualmente fundidas nas superfícies

Tabela 1. Dados morfométricos dos esporos de Polytrichaceae (unidades em $\mu \mathrm{m})$. (DP $=$ diâmetro polar; $\mathrm{DE}=$ diâmetro equatorial).

Table 1. Polytrichaceae spores morphometric data (units in $\mu \mathrm{m}) .(\mathrm{DP}=$ polar diameter; $\mathrm{DE}=$ equatorial diameter).

\begin{tabular}{|c|c|c|c|c|c|c|}
\hline \multirow{2}{*}{$\begin{array}{l}\text { Espécies } \\
\text { Coletor } \mathrm{n}^{\mathrm{o}}\left(\mathrm{n}^{\mathrm{o}} \text { coleção) }\right.\end{array}$} & \multirow{2}{*}{$\begin{array}{r}\mathrm{DP} \\
\bar{x}\end{array}$} & \multirow{2}{*}{$\frac{\mathrm{DE}}{\bar{x}}$} & \multicolumn{4}{|c|}{ Diâmetro Maior } \\
\hline & & & $\left(x_{\text {min }}-x_{\text {máx }}\right) \bar{x} \pm s_{x}$ & $s$ & $95 \% I C$ & $C V(\%)$ \\
\hline
\end{tabular}

Atrichum androgynum (Müll. Hal.)

A. Jaeger

A. Sehnem 83016

$10,03 \quad 15,06$

$(12,76-15,84) 14,96 \pm 0,07$

0,66

14,83-15,09

4,41

A. Sehnem 83004

$9,75 \quad 14,89$

$(13,64-15,84) \quad 14,90 \pm 0,06$

0,64

$14,77-15,03$

4,30

Itatiella ulei (Broth ex Müll. Hal.)

G. L. Sm.

O. Yano s.n. (RB210623)

D.M. Vital \& W. R. Buck 11764

D. M. Vital 3631

O. Yano \& Z. R. de Melo 22539

\begin{tabular}{|c|c|c|c|c|c|}
\hline 20,05 & 30,88 & $(28,75-32,00)$ & $30,23 \pm 0,13$ & 0,71 & $29,97-30,49$ \\
\hline 20,00 & 28,08 & $(27,00-30,50)$ & $28,80 \pm 0,22$ & 1,20 & $28,35-29,25$ \\
\hline 18,26 & 25,72 & $(18,75-30,00)$ & $26,61 \pm 0,17$ & 1,67 & $26,28-26,94$ \\
\hline 19,88 & 28,51 & $(27,50-32,50)$ & $29,29 \pm 0,11$ & 1,15 & $29,06-29,52$ \\
\hline 16,24 & 21,03 & $(18,70-23,76)$ & $21,17 \pm 0,11$ & 1,13 & $20,95-21,39$ \\
\hline 16,08 & 23,69 & $(22,50-26,25)$ & $23,51 \pm 0,10$ & 1,04 & $23,30-23,72$ \\
\hline 19,70 & 28,95 & $(26,25-30,50)$ & $28,68 \pm 0,21$ & 1,17 & $28,24-29,12$ \\
\hline 9,9 & 29,20 & $(27,00-30,50)$ & $29,04 \pm 0$ & 1,01 & $28,66-29,42$ \\
\hline & 25,35 & $(23,00-27,50)$ & $25,10 \pm 0,09$ & 0,94 & $24,91-25,29$ \\
\hline 18,61 & 25,75 & $(23,00-27,50)$ & $25,36 \pm 0,11$ & 1,12 & $25,14-25,58$ \\
\hline
\end{tabular}

Oligotrichum denudatum Merril Vital \& Buck V 11941

Oligotrichum riedelianum (Mont.) Mitt. P. Dusén 493

Pedro Occhioni s.n. (RB 218309)

Fidalgo \& Kauffman Fidalgo $E g-07$ (RB)

O. Yano \& Z. R. de Melo 22628

A. Sehnem 83017

$18,61 \quad 25,75$

$(23,00-27,50) 25,36 \pm 0,11$

1,12

3,74 
continuação

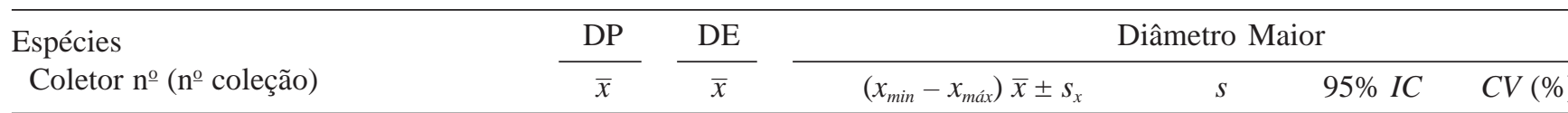

Pogonatum brasiliense (Hampe)

A. Jaeger

J. M. A. Braga et. al. 5252

$6,77 \quad 9,01 \quad(8,36-9,90) \quad 8,94 \pm 0,03 \quad 0,28 \quad 8,88-9,00 \quad 3,13$

Pogonatum campylocarpum

(Müll. Hal.) Mitt.

Löfgren \& Edwall 537

G. Martinelli et al. 2460

Brotherus s.n. (RB218294)

\begin{tabular}{|c|c|c|c|c|}
\hline & 14,66 & $(14,50-16,25) 14,91 \pm 0,04$ & 0,37 & $14,84-14,98$ \\
\hline 41 & 11,35 & $(10,56-12,10) 11,13 \pm 0,03$ & 0,32 & $11,07-11,19$ \\
\hline 8,55 & 11,22 & $(10,56-13,20) \quad 11,41 \pm 0,05$ & 0,46 & $11,32-11,50$ \\
\hline
\end{tabular}

Pogonatum pensilvanicum (Müll. Hal.)

Mitt.
L. Krieger 13949
D. M. Vital 1236
D. M. Vital 8856
O. Yano \& J. R. Pirani 7298

$\begin{array}{rr}11,16 & 15,03 \\ 10,62 & 15,15 \\ 9,23 & 14,75 \\ 9,57 & 13,75\end{array}$

$(13,00-17,00) 15,16 \pm 0,07$

0,67

$15,03-15,29$

4,42

$(13,75-16,25) 15,07 \pm 0,11$

0,58

$14,85-15,29$

3,85

$(13,00-15,50) 14,72 \pm 0,13$

0,70

$14,46-14,98$

4,75

$(13,00-17,00) \quad 15,16 \pm 0,07$

0,67

$15,03-15,29$

4,42

Pogonatum tortile (Sw.) Brid.

E. Ule 1319

Brotherus s.n. (RB218302)

$7,46 \quad 11,20$

$(9,90-12,10) \quad 11,22 \pm 0,08 \quad 0,45$

$11,05-11,39$

4,10

$8,76 \quad 11,10$

$(9,90-11,44) \quad 10,99 \pm 0,04$

0,36

$10,92-11,06$

3,28

Polytrichadelphus pseudopolytrichum

(Raddi) G. L. Sm.

O. Yano 23875

T. Vianna s.n. (BHCB2005)

A. Sehnem 83028

E. Ule s.n. (R82777)

L. Nette s.n. (R14259)

E. Ule 11314

E. Ule 1314

$\begin{array}{rr}8,41 & 11,17 \\ 5,44 & 7,73 \\ 8,46 & 11,43 \\ 8,83 & 12,03 \\ 6,05 & 8,08 \\ 8,35 & 10,82 \\ 10,04 & 12,19\end{array}$

$(10-12,5) \quad 11,15 \pm 0,07$

$(7-9,5) \quad 7,84 \pm 0,04$

$(10,5-12,5) \quad 11,59 \pm 0,06$

$(10,5-12,5) \quad 12,22 \pm 0,04$

$(7,5-9,5) \quad 7,97 \pm 0,06$

$(10-12,5) \quad 11,02 \pm 0,08$

$(10,5-13,75) \quad 12,43 \pm 0,05$

0,68

$11,02-11,28$

6,10

$0,41 \quad 7,76-7,92 \quad 5,23$

$0,65 \quad 11,46-11,72 \quad 5,60$

$0,45 \quad 12,13-12,31 \quad 3,68$

$\begin{array}{lll}0,58 & 7,85-8,09 & 7,28\end{array}$

Polytrichum commune L. ex Hedw.

G. Hatschbach 14136

Kummrow 826

Schwacke s.n. (R14260)

Rangel \& Schwacke s.n. (R14257)

Warmstorf s.n. (R82772)

$9,01 \quad 13,34$

$9,56 \quad 13,53$

$(12,00-15,00) 13,32 \pm 0,09$

0,78

$10,87-11,17 \quad 7,08$

$7,51 \quad 9,28$

$8,45 \quad 11,44$

$(12,50-15,00) \quad 13,47 \pm 0,08$

$(11,25-13,75) \quad 12,56 \pm 0,04$

$(8,00-10,50) \quad 9,41 \pm 0,07$

0,51

$12,34-12,54$

4,10

$6,51 \quad 9,74$

$(8,80-10,56) \quad 9,62 \pm 0,10$

0,89

$13,14-13,50$

6,68

$0,78 \quad 13,32-13,62 \quad 5,79$

$0,44 \quad 12,47-12,65 \quad 3,50$

$0,70 \quad 9,27-9,55 \quad 7,44$

Polytrichum juniperinum var.

juniperinum Hedw.

O. Yano \& P. I. S. Braga 10491

O. Yano \& D. P. Santos 6142

J. Vidal s.n. (R87685)

Luiz E. M. F. 3437

E. Pereira 2638 \& Pabst 3474

P. M. Andrade s.n. (BHCB23892)

$8,06 \quad 11,53$

$8,40 \quad 11,28$

$7,72 \quad 10,35$

$7,90 \quad 10,20$

$8,00 \quad 10,48$

$7,45 \quad 10,25$

$(10,00-12,50) \quad 11,25 \pm 0,08$

$(10,50-12,50) \quad 11,32 \pm 0,12$

$(10,00-11,25) \quad 10,45 \pm 0,08$

$(10,00-11,25) \quad 10,21 \pm 0,06$

$(10,00-12,00) 10,41 \pm 0,10$

$(10,00-11,25) 10,38 \pm 0,06$

0,75

0,65

0,44

0,31

0,55

0,30

$11,10-11,40$

6,67

$11,09-11,57$

5,74

$10,27-10,59 \quad 4,21$

$10,09-10,33 \quad 3,04$

$10,20-10,62 \quad 5,28$

$10,07-10,29 \quad 2,89$

Polytrichum juniperinum var. paulense

(Geh. \& Hampe) Herzog.

E. Ule s.n. (MG20122)

A. Sehnem 9998

$7,50 \quad 10,82$

$7,06 \quad 9,77$

$(9,24-12,10) \quad 10,60 \pm 0,07$

$(8,80-11,00) \quad 9,74 \pm 0,12$

0,67

0,66

$10,47-10,73$

6,32

$9,49-9,99 \quad 6,78$

Polytrichum subremotifolium Geh.

\& Hampe

G. Hatschbach 19527

$8,65 \quad 10,90$

$(9,90-11,44) \quad 10,87 \pm 0,03$

0,35

$10,80-10,94$

3,22 

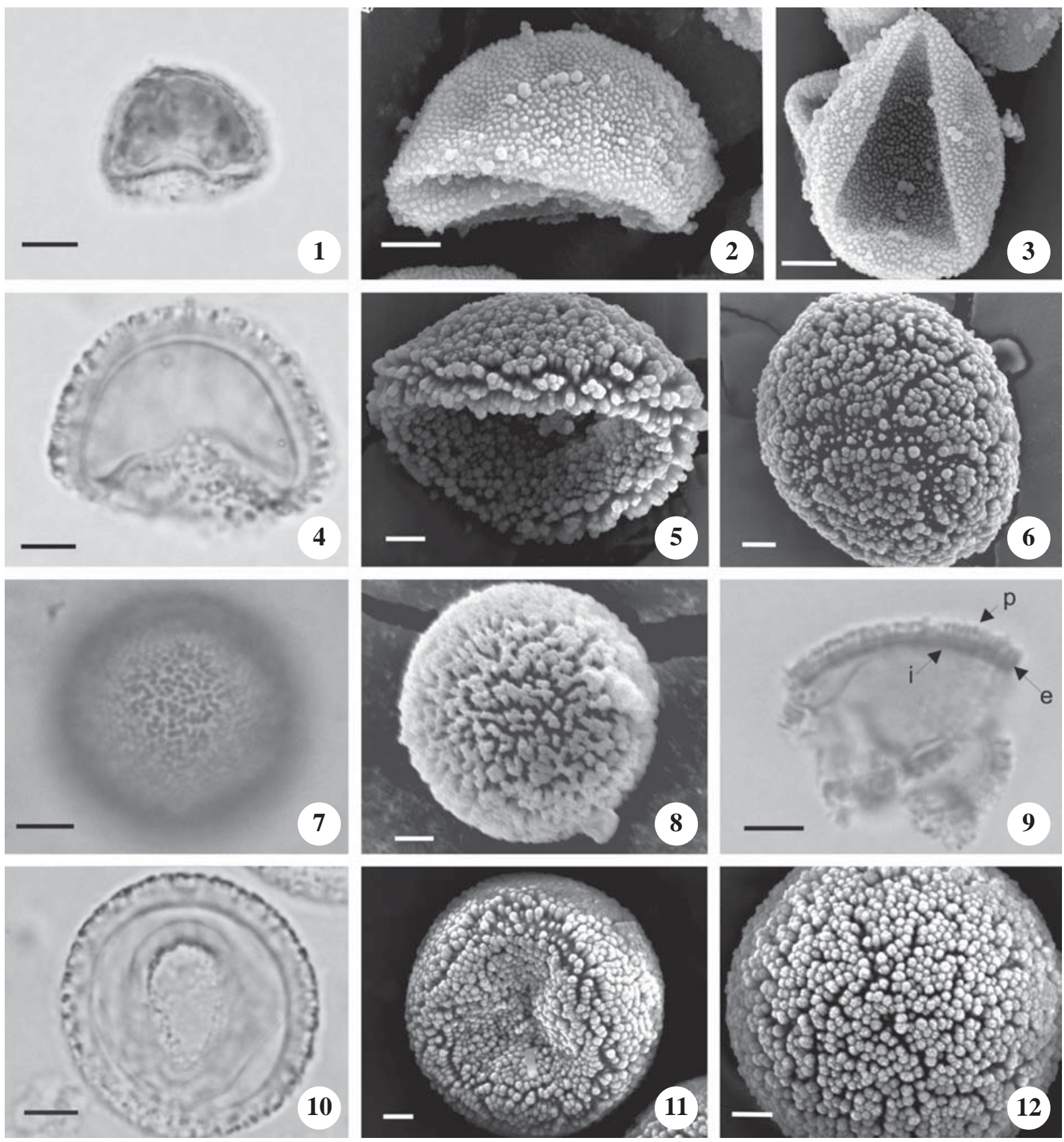

Figuras 1-12. Fotomicrografias de microscopia de luz e eletrônica de varredura (MEV) dos esporos de Polytrichaceae. 1-3. Atrichum androgynum. 1. Vista equatorial, corte óptico, gelatina corada com verde metila. 2. Vista equatorial, MEV. 3. Vista polar proximal, abertura, MEV. 4-6. Itatiella ulei. 4. Vista equatorial, corte óptico, acetólise. 5. Vista equatorial, MEV. 6. Vista polar distal, MEV. 7-9. Oligotrichum denudatum. 7. Vista polar distal, superfície, gelatina corada com fucsina básica. 8. Vista polar distal, MEV. 9. Camadas do esporoderma (setas). 10-12. Oligotrichum riedelianum. 10. Vista polar proximal, abertura, acetólise. 11. Vista polar proximal, abertura, MEV. 12. Vista polar distal, MEV. Barra $=5 \mu \mathrm{m}(1,4,7$, 9-10); 3 нm (2-3-5-6-8, 11-12).

Figures 1-12. Light photomicrographs and scanning electronmicrographs (SEM) of Polytrichaceae spores. 1-3. Atrichum androgynum. 1. Equatorial view, optical section, glycerin jelly stained with methyl green. 2. Equatorial view, SEM. 3. Proximal view, aperture, SEM. 4-6. Itatiella ulei. 4. Equatorial view, optical section, acetolysis. 5. Equatorial view, SEM. 6. Distal view, SEM. 7-9. Oligotrichum denudatum. 7. Distal view, surface, glycerin jelly stained with basic fucsin. 8. Distal view, SEM. 9. Sporoderm layers (arrows). 10-12. Oligotrichum riedelianum. 10. Proximal view, aperture, acetolysis. 11. Proximal view, aperture, SEM. 12. Distal view, SEM. Bar $=5 \mu \mathrm{m}(1,4,7,9-10) ; 3 \mu \mathrm{m}(2-3-5-6-8,11-12)$. 
proximal e distal (figuras 2-3), e grânulos regulares, isolados ou fundidos também nas duas faces.

Exina psilada, com espessura não uniforme, mais espessa no pólo distal (tabela 2).

Intina mais espessa na região proximal (figura 1 , tabela 2).

A presença de uma região apertural na face proximal dos esporos é caracterizada pelas variações na espessura da intina e da exina, e pela clara definição heteropolar dada pela forma dos esporos (tabela 2).

Itatiella G. L. Sm.

Itatiella ulei (Broth. ex Müll. Hal.) G. L. Sm.

Esporos isomórficos, de tamanho médio (tabela 1), heteropolares, âmbito subcircular, com região apertural proximal, superfície formada por elementos alongados, fundidos ou isolados, por grânulos e pilos (figuras 4-6).

A região apertural possui forma variável, podendo se apresentar sob a forma subcircular (figura 5), triangular ou ainda irregular.

Sob ML, observa-se apenas o ápice dos elementos de ornamentação da perina e a fusão de alguns destes elementos na base. Em vista equatorial pode-se observar o tamanho e o tipo dos elementos presentes na face distal e proximal. Sob MEV percebem-se elementos alongados fundidos na base ou isolados, pilos e báculos na face distal (figura 5-6).

Exina psilada com espessura não uniforme, menos espessa no pólo proximal (figura 4, tabela 2).

Intina mais espessa na região proximal (figura 4, tabela 2).

Tabela 2. Dados morfométricos do esporoderma dos esporos de Polytrichaceae (unidades em $\mu \mathrm{m}$ ) $n=10$.

Table 2. Sporoderm of Polytrichaceae spore morphometric data (units in $\mu \mathrm{m}) n=10$.

\begin{tabular}{|c|c|c|c|c|}
\hline \multirow[b]{2}{*}{ Espécies } & \multicolumn{3}{|c|}{ Wodehouse } & \multirow{2}{*}{$\begin{array}{c}\text { Acetólise } \\
\text { Esclerina } \\
\text { distal total }\end{array}$} \\
\hline & $\begin{array}{l}\text { Intina } \\
\text { proximal }\end{array}$ & $\begin{array}{l}\text { Intina } \\
\text { distal }\end{array}$ & $\begin{array}{l}\text { Esclerina } \\
\text { distal total }\end{array}$ & \\
\hline $\begin{array}{l}\text { Atrichum androgynum (Müll. Hal.) A. Jaeger } \\
\text { A. Sehnem } 83016\end{array}$ & 1,10 & 0,37 & 1,14 & 1,28 \\
\hline $\begin{array}{l}\text { Itatiella ulei (Broth. ex Müll. Hal.) G. L. Sm. } \\
\text { D. M. Vital } 3631\end{array}$ & 1,94 & 0,81 & 2,29 & 2,49 \\
\hline $\begin{array}{l}\text { Oligotrichum denudatum Merril } \\
\text { Vital \& Buck V } 11941\end{array}$ & 2,38 & 1,83 & 2,24 & 2,42 \\
\hline $\begin{array}{l}\text { Oligotrichum riedelianum (Mont.) Mitt. } \\
\text { P. Dusén } 493\end{array}$ & 1,89 & 0,97 & 3,67 & 4,58 \\
\hline $\begin{array}{l}\text { Pogonatum brasiliense (Hampe) A. Jaeger } \\
\text { J. M. A. Braga et al. } 5252\end{array}$ & 0,81 & 0,33 & 1,01 & 0,88 \\
\hline $\begin{array}{l}\text { Pogonatum campylocarpum (Müll. Hal.) Mitt. } \\
\text { Löfgren \& Edwall } 537\end{array}$ & 1,28 & 042 & 1,14 & 1,01 \\
\hline $\begin{array}{l}\text { Pogonatum pensilvanicum (Müll. Hal.) Mitt. } \\
\text { L. Krieger } 13949\end{array}$ & 0,90 & 0,35 & 0,92 & 1,01 \\
\hline $\begin{array}{l}\text { Pogonatum tortile (Sw.) Brid. } \\
\text { Brotherus s.n. }(\mathrm{RB} 218302)\end{array}$ & 0,92 & 0,37 & 1,14 & 1,23 \\
\hline $\begin{array}{l}\text { Polytrichadelphus pseudopolytrichum (Raddi) G. L. Sm. } \\
\text { T. Vianna s.n. (BHCB2005) }\end{array}$ & 0,64 & 0,22 & 0,68 & 0,77 \\
\hline $\begin{array}{l}\text { Polytrichum commune L. ex Hedw. } \\
\text { Rangel et Schwacke s.n. (R 14257) }\end{array}$ & 0,68 & 0,22 & 0,79 & 0,84 \\
\hline $\begin{array}{l}\text { Polytrichum juniperinum var. juniperinum Hedw. } \\
\text { O. Yano \& P. I. S. Braga } 10491\end{array}$ & 0,81 & 0,35 & 0,99 & 1,12 \\
\hline $\begin{array}{l}\text { Polytrichum juniperinum var. paulense (Geh. \& Hampe) } \\
\text { Herzog }\end{array}$ & & & & \\
\hline E. Ule s.n. (MG 20122) & 0,90 & 0,42 & 1,25 & 0,70 \\
\hline $\begin{array}{l}\text { Polytrichum subremotifolium Geh. \& Hampe } \\
\text { G. Hatschbach } 19527\end{array}$ & 0,73 & 0,31 & 0,79 & 0,70 \\
\hline
\end{tabular}


A caracterização da região apertural se deu pela ornamentação bastante irregular na face proximal e regular na face distal.

Oligotrichum Lam. \& DC.

Oligotrichum denudatum Merril

Oligotrichum riedelianum (Mont.) Mitt.

Esporos isomórficos, de tamanho pequeno a médio (tabela 1), heteropolares, âmbito subcircular, com região apertural proximal, superfície formada por báculos isolados, verrucosos no ápice, eventualmente fundidos no ápice, pilos e clavas isolados na face proximal e processos alongados ornamentados (figuras 7-12).

A região apertural é facilmente visualizada, com forma variando de subtriangular a arredondada (figuras 10-11).

Elementos de ornamentação facilmente visualizados em ML, mas melhor evidenciados em MEV. Em vista polar proximal pode-se perceber a diferença de distribuição dos elementos de ornamentação e sua disposição delimitando a região apertural (figuras 8, 11-12).

Exina psilada, com espessura não uniforme, menos espessa no pólo proximal (tabela 2).

Intina bem espessa na região proximal (tabela 2).

Pogonatum P. Beauv.

Pogonatum brasiliense (Hampe) A. Jaeger

Pogonatum campylocarpum (Müll. Hal.) Mitt.

Pogonatum pensilvanicum (Hedw.) P. Beauv.

Pogonatum tortile (Sw.) Brid.

Esporos isomórficos, de tamanho muito pequeno (tabela 1), heteropolares, âmbito subcircular, com região apertural proximal, superfície variável nas diferentes espécies, formada por pequenas projeções cônicas a grânulos isolados na face distal e, eventualmente, áreas lisas (figuras 13-24).

A região apertural possui forma variando de triangular a irregular. Não é facilmente visualizada, porém há alterações na distribuição dos elementos de ornamentação (figuras 14, 19, 22-23).

Elementos de ornamentação muito reduzidos e de difícil visualização sob ML (figuras 17-18). Sob MEV, a ornamentação pode ser caracterizada pela presença de elementos em forma de projeções cônicas (em Pogonatum brasiliense, figuras 13-15), grânulos isolados, ornamentados ou lisos (em Pogonatum campylocarpum, figura 16), grânulos irregulares, isolados ou unidos (em Pogonatum pensilvanicum, figuras 17-20) e grânulos associados a gemas isoladas e discretas áreas psiladas (em Pogonatum tortile, figuras 21-24).
Exina psilada, com espessura não uniforme, menos espessada na região proximal do esporo (tabela 2).

Intina mais espessa na região proximal (figuras 13 , 17-18, tabela 2).

Polytrichadelphus (Müll. Hal.) Mitt. Polytrichadelphus pseudopolytrichum (Raddi) G. L. Sm.

Esporos isomórficos, de tamanho muito pequeno a pequeno (tabela 1), heteropolares, âmbito subcircular, com região apertural proximal, superfície formada por pequenos espinhos lisos, regularmente distribuídas pelos pólos distal e proximal (figura 26).

A região apertural apresenta forma subtriangular, sendo distinta sob ML pela diferença na espessura da intina proximal (figura 26).

Elementos de ornamentação pequenos e de visualização parcial em ML. Sob MEV a ornamentação mostra-se regular. Na região distal e na proximal, ocorre uma distribuição regular de pequenos espinhos lisos por toda a superfície do esporo. Algumas áreas lisas também são encontradas, evidenciando a exina não ornamentada.

Exina psilada, menos espessa na região proximal (tabela 2).

Intina apresentando um grande espessamento na região proximal (tabela 2), local onde ocorre a germinação (figura 25).

Polytrichum L. ex Hedw.

Polytrichum commune L. ex Hedw.

Polytrichum juniperinum var. juniperinum Hedw.

Polytrichum juniperinum var. paulense (Geh. \& Hampe) Herzog

Polytrichum subremotifolium Geh. \& Hampe

Esporos isomórficos, de tamanho pequeno (tabela 1), heteropolares, âmbito subcircular, com região apertural proximal, superfície formada por processos espinhosos ornamentados a grânulos regulares, isolados ou unidos, distribuídos pelos pólos distal e proximal de maneira regular ou irregular, nas diferentes espécies estudadas (figuras 27-36).

A região apertural apresenta-se com forma irregular variando de sub-triangular, sub-circular ou alongada (figuras 28, 35). A distinção desta região pode ser realizada pelo espessamento proximal da intina.

A ornamentação é distinta nas diferentes espécies, varia de processos espinhosos ornamentados (em Polytrichum commune, figuras 27-28), grânulos regulares (em Polytrichum juniperinum var. juniperinum, figuras 29-30), grânulos irregulares isolados ou unidos (em 

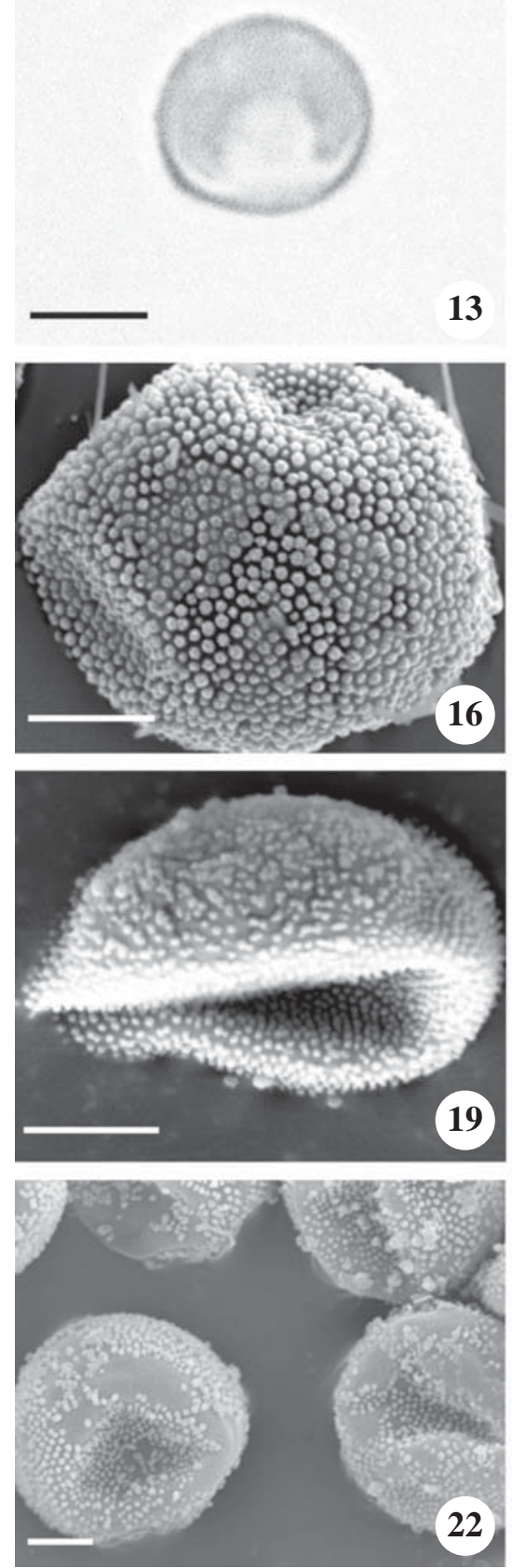
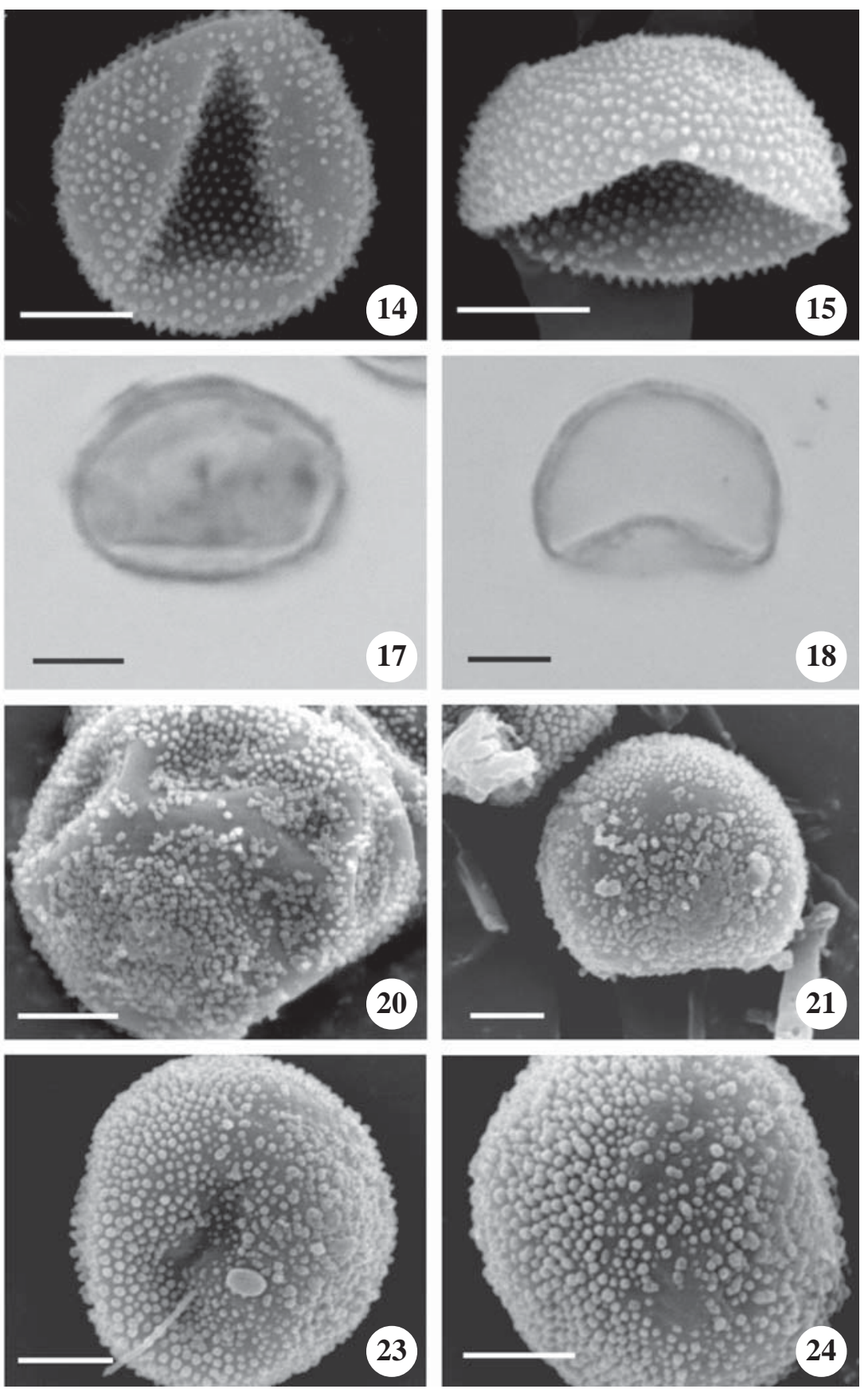

Figuras 13-24. Fotomicrografias de microscopia de luz e eletrônica de varredura dos esporos de Polytrichaceae. 13-15. Pogonatum brasilisense. 13. Vista equatorial, corte óptico, gelatina corada com verde metila. 14. Vista polar proximal, abertura, MEV. 15. Vista equatorial, MEV. 16. Pogonatum campylocarpum vista polar distal, MEV. 17-20. Pogonatum pensilvanicum. 17. Vista equatorial, corte óptico, gelatina corada com verde metila. 18. Vista equatorial, corte óptico, acetólise. 19. Vista equatorial, MEV. 20. Vista polar distal, MEV. 21-24. Pogonatum tortile. 21. Vista equatorial, MEV. 22. Vista geral, MEV. 23. Vista polar proximal, MEV. 24. Vista polar distal, MEV. Barra $=5 \mu \mathrm{m}(13,17-18) ; 3 \mu \mathrm{m}(14-16,19-24)$.

Figures 13-24. Light photomicrographs and scanning electronmicrographs of Polytrichaceae spores. 13-15. Pogonatum brasilisense. 13. Equatorial view, optical section, glycerin jelly stained with methyl green. 14. Proximal view, aperture, SEM. 15. Equatorial view, SEM. Fig. 16. Pogonatum campylocarpum: distal view, MEV. 17-20. Pogonatum pensilvanicum. 17. Equatorial view, optical section, glycerin jelly stained with methyl green. 18. Equatorial view, optical section, acetolysis. 19. Equatorial view, SEM. 20. Distal view, SEM. 21-24. Pogonatum tortile. 21. Equatorial view, SEM. 22. General view, SEM. 23. Proximal view, SEM. 24. Distal view, SEM. Bar $=5 \mu \mathrm{m}(13,17-18) ; 3 \mu \mathrm{m}(14-16,19-24)$. 


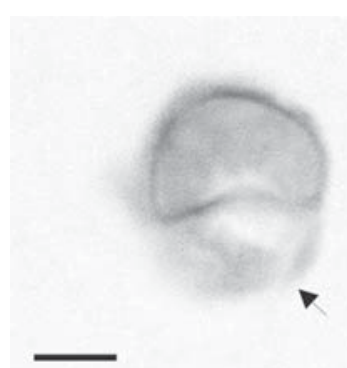

25
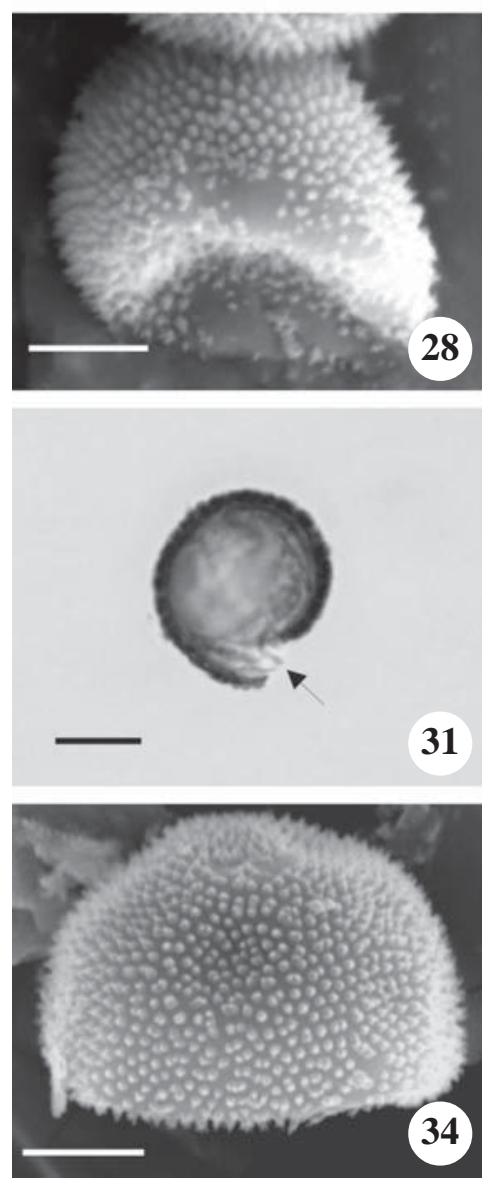
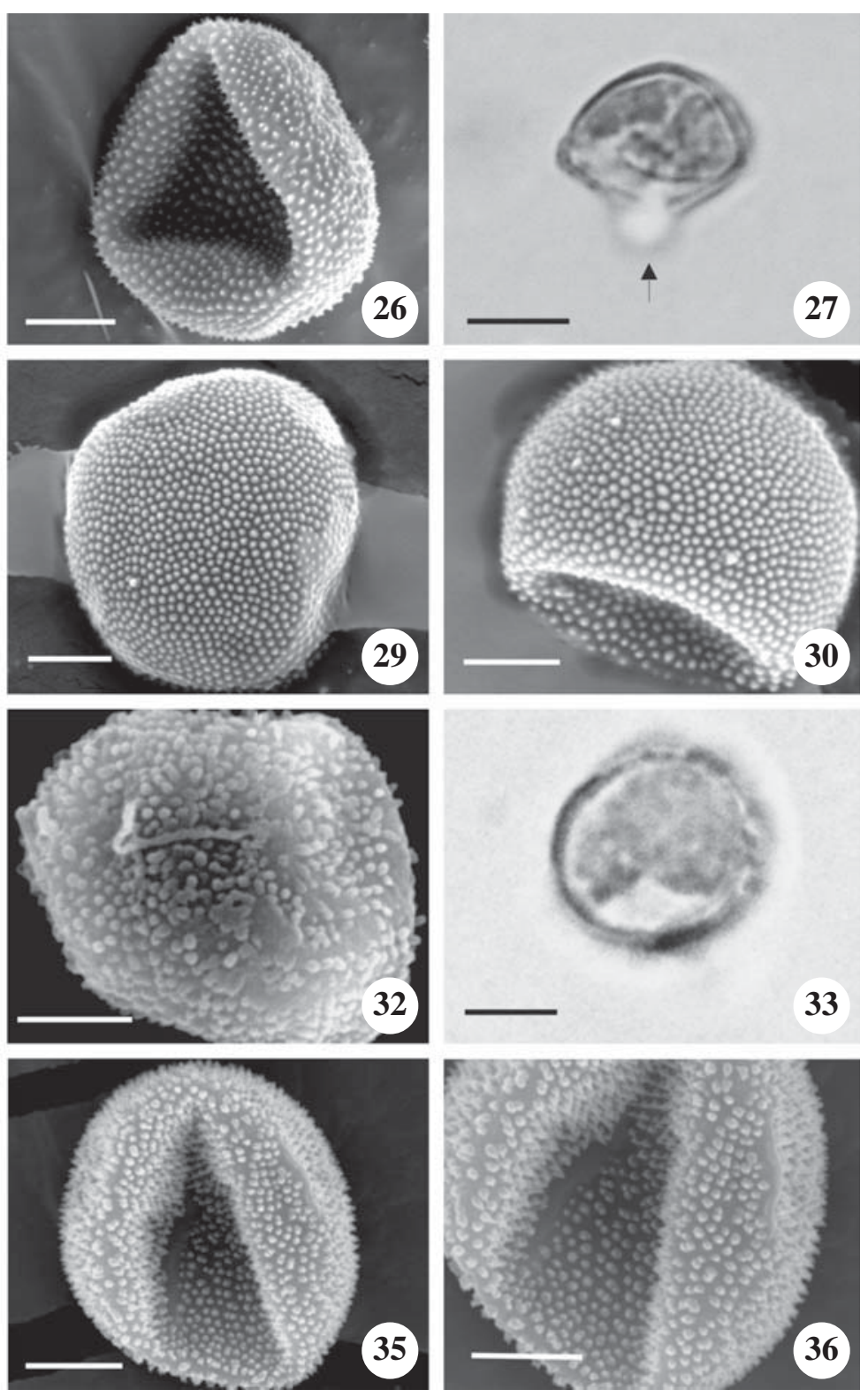

Figuras 25-36. Fotomicrografias de microscopia de luz e eletrônica de varredura dos esporos de Polytrichaceae. 25-26. Polytrichadelphus pseudopolytrichum. 25. Vista equatorial, esporo liberando conteúdo celular, corado com fucsina básica (seta). 26. Vista polar proximal, MEV. 27-28. Polytrichum commune. 27. Vista equatorial, esporo em germinação corado com verde de metila (seta). 28. Vista equatorial, MEV. 29-30. Polytrichum juniperinum var. juniperinum. 29. Vista polar distal, MEV. 30. Vista equatorial, MEV. 31-32. Polytrichum juniperinum var. paulense. 31. Vista equatorial, esporo liberando conteúdo celular, corado com verde metila (seta). 32. Vista polar distal, MEV. 33-36. Polytrichum subremotifolium. 33. Vista equatorial, corte óptico, gelatina corada com verde metila. 34. Vista equatorial, MEV. 35. Vista polar proximal, MEV. 36. Vista polar proximal, MEV, detalhe. Barra $=5 \mu \mathrm{m}(25,27,31$ e 33$) ; 3 \mu \mathrm{m}(26,28-30,32,34-36)$.

Figures 25-36. Light photomicrographs and scanning electronmicrographs of Polytrichaceae spores. 25-26. Polytrichadelphus pseudopolytrichum. 25. Equatorial view, germinating spore satined with basic fucsin (arrow). 26. Proximal view, SEM. 27-28. Polytrichum commune. 27. Equatorial view, germinating spore stained with methyl green (arrow). 28. Equatorial view, SEM. 29-30. Polytrichum juniperinum var. juniperinum. 29. Distal view, SEM. 30. Equatorial view, SEM. 31-32. Polytrichum juniperinum var. paulense. 31. Equatorial view, germinating spore stained with methyl green (arrow). 32. Distal view, SEM. 33-36. Polytrichum subremotifolium. 33. Equatorial view, optical section, glycerin jelly stained with methyl green. 34. Equatorial view, SEM. 35. Proximal view, SEM. 36. Proximal view, SEM, detail. Bar $=5 \mu \mathrm{m}(25,27,31$ and 33$) ; 3 \mu \mathrm{m}$ $(26,28-30,32,34-36)$. 
Polytrichum juniperinum var. paulense, figuras 31-32) e projeções cônicas com ápice irregular (em Polytrichum subremotifolium, figuras 33-36). Áreas lisas também são eventualmente encontradas, principalmente, na região equatorial (figura 28 ).

Exina psilada, com espessura reduzida na região proximal (tabela 2).

Intina mais espessa na região proximal (figuras 27 , 31-33, tabela 2).

\section{Discussão}

Foi possível observar que a ornamentação dos esporos de Polytrichaceae é formada por elementos da perina sob a forma de grânulos unidos ou isolados, gemas isoladas, clavas, báculos livres ou fundidos, pilos isolados, projeções cônicas ou ainda processos espinhosos ornamentados.

Os esporos de todas as espécies estudadas são isomórficos; alguns sem conteúdo citoplasmático (abortados) foram encontrados, estes ocorreram em maior quantidade nas amostras das espécies Atrichum androgynum, Itatiella ulei e Oligotrichum riedelianum. A presença de esporos abortados em musgos é uma característica comum ao grupo (Olesen \& Mogensen 1978).

A caracterização da ornamentação da superfície foi complementada com observações sob microscopia eletrônica de varredura, que revelou uma variabilidade dos elementos de ornamentação permitindo caracterizar espécies ou grupos de espécies.

O levantamento de literatura realizado demonstra o número reduzido de estudos descrevendo esporos de Polytrichaceae. McClymont \& Larson (1964), Nyholm (1971), Smith (1971) e Stefureac et al. (1979) empregaram terminologias diferentes para caracterizar os esporos, porém, é possível constatar que as descrições apresentadas para os esporos de Polytrichum commune e Atrichum androgynum são aqui corroboradas, o que não acontece com Polytrichum juniperinum var. juniperinum, uma vez que os resultados obtidos no presente trabalho demonstram que a superfície dos esporos da espécie é granulada.

Mullen \& Frye (1947) consideraram os esporos de Oligotrichum riedelianum como quase lisos e de tamanho pequeno. Os dados aqui obtidos demonstram que a espécie possui esporos com ornamentação muito elaborada, apresentando báculos, clavas e pilos alongados. A amplitude das médias de tamanho dos diâmetros demonstra que os esporos de Oligotrichum riedelianum são de tamanho médio.
As observações de Erdtman $(1957,1965)$ sobre a ornamentação da superfície, forma e estratificação do esporoderma dos esporos dos gêneros Atrichum, Pogonatum e Polytrichum foram confirmadas pelos resultados obtidos no presente trabalho.

Comparando-se os presentes resultados com aqueles obtidos por Smith (1974), nota-se que a grande diferença na terminologia empregada para descrever os esporos impede corroborar os dados daquele autor. Foi possível observar que esporos de Oligotrichum riedelianum apresentam báculos, pilos e clavas isolados ou, eventualmente, fundidos, e esporos de Polytrichum commune possuem processos espinhosos ornamentados.

Farias (1984) ao estudar, exclusivamente sob microscopia eletrônica de varredura, os esporos de sete espécies ocorrentes no Brasil, encontrou resultados que são aqui corroborados, exceto para Polytrichum juniperinum var. juniperinum nos quais o tipo de ornamentação descrito não evidencia o padrão granulado observado no presente trabalho. No entanto, a terminologia adotada naquele trabalho não está de acordo com o padrão adotado em palinologia atualmente.

Hyvönen et al. (1998) incluíram a ornamentação da superfície do esporo como um dos caracteres morfológicos na análise filogenética de Polytrichales, entretanto, os autores trataram tal caráter como possuindo apenas quatro estados: esporos papilosos, espiculosos, tipo Bartramiopsis e tipo Oedipodium. No presente trabalho, foi possível caracterizar um maior número de tipos palinológicos o que possibilitou referendar a separação genérica proposta por Smith $(1971,1974)$ e Derda et al. (1999).

As características dos elementos de ornamentação dos esporos das espécies estudadas permitem inferir dois grupos afins, um deles incluindo os esporos de Itatiella, Oligotrichum, Atrichum e Pogonatum, e outro, representado pelos esporos de Polytrichadelphus e Polytrichum.

Polytrichum juniperinum var. paulense e Polytrichum subremotifolium são consideradas espécies duvidosas, porém, são nomes considerados válidos, de acordo com os dados do $\mathrm{W}^{3}$ Most (Moss Database Nomeclature (2006)). Dados aqui observados adicionam informações importantes para a interpretação futura de tais espécies.

Os esporos de Polytrichum juniperinum var. paulense possuem ornamentação granulada bastante distinta da observada nos esporos da outra variedade da espécie analisada (Polytrichum juniperinum var. juniperinum).

Os esporos de Polytrichum subremotifolium apresentam superfície com projeções espinhosas ornamentadas e amplitude das médias dos diâmetros 
semelhantes às observadas em esporos de Polytrichum commune e Pogonatum brasiliense.

Pogonatum brasiliense é outra espécie que merece destaque por possuir esporos de tamanho muito pequeno e superfície formada por projeções cônicas, características próximas àquelas encontradas em esporos de espécies de Polytrichum. Tais feições permitem considerar a afirmação de Hyvönen (1989) de que Pogonatum brasiliense deveria permanecer no gênero Polytrichum, assim como foi, originalmente nomeada por Hampe (1872).

Estudos sobre morfologia de esporos de Oligotrichum denudatum, Pogonatum tortile, Polytrichum juniperinum var. paulense e Polytrichum subremotifolium não foram encontrados na literatura consultada.

A morfologia dos esporos da família Polytrichaceae mostra aspectos de importância taxonômica.

As espécies estudadas no presente trabalho apresentaram esporos isomórficos, de tamanho muito pequeno a médio, heteropolares, com região apertural subtriangular ou arredondada e esporoderma composto por intina, exina e perina. Estas são características típicas dos esporos de Bryophyta.

A ornamentação da perina dos esporos variou de granulada (Atrichum androgynum, Pogonatum campylocarpum, Pogonatum pensilvanicum, Pogonatum tortile, Polytrichum juniperinum var. juniperinum e Polytrichum juniperinum var. paulense), pilada, com báculos e clavas isoladas (Itatiella ulei, Oligotrichum riedelianum e Oligotrichum denudatum), com espinhos lisos (Polytrichadelphus pseudopolytrichum), processos espinhosos ornamentados (Polytrichum commune) e projeções cônicas (irregulares em Polytrichum subremotifolium e regulares em Pogonatum brasiliense).

Agradecimentos - Os autores agradecem a todos aqueles que contribuíram para a realização deste trabalho, especialmente, à Dra. Olga Yano, pelas valiosas sugestões, aos curadores dos herbários (BHCB, CESJ, MG, HB, PACA, R, RB e SP) e aos centros de microscopia eletrônica da UFRJ e da USU. $\mathrm{O}$ primeiro autor agradece à CAPES, Coordenação do Aperfeiçoamento do Pessoal do Nível Superior, pela bolsa concedida durante a realização da dissertação. A segunda autora agradece ao $\mathrm{CNPq}$, Conselho Nacional de Desenvolvimento Científico e Tecnológico, (Proc.481595/ 2004-0) pela bolsa de produtividade. A terceira autora agradece à FAPEMIG, Fundação de Amparo à Pesquisa do Estado de Minas Gerais, pelo apoio financeiro concedido (2772/98), favorecendo esta linha de pesquisa na UFJF.

\section{Referências bibliográficas}

BARTH, O.M. \& MELHEM, T.S. 1988. Glossário ilustrado de palinologia. Editora Unicamp, Campinas.
BOVE, C.P. 2000. Phylogenetic analysis of Humiriaceae with notes on the monophyly of Ixonanthaceae. Journal Complishment Biology 2:19-24.

BOVE, C.P. \& MELHEM, T.S. 2000. Humiriaceae. World Pollen and Spore Flora 22:1-35.

BUCK, W.R. \& GOFFINET, B. 2000. Morphology and classification of mosses. In Bryophyte Biology (J.A. Shaw \& B. Goffinet, eds.). Cambridge University Press, Cambridge, p.71-123.

CRUM, H. 1976. Mosses of the Great Lakes Forest. Ed. University of Michigan Herbarium, Ann Arbor.

DERDA, G.S., WYATT, R. \& HYVÖNEN, J. 1999. Genetic similarities among the hair-cap mosses (Polytrichaceae) as revealed by enzyme eletrophoresis. The Bryologist 102:352-365.

ERDTMAN, G. 1957. Pollen and spore morphology/ plant taxonomy. Gymnospermae, Pteridophyta, Bryophyta (Illustrations). An introduction to palynology II. The Chronica Botanic Company, Stockholm.

ERTDMAN, G. 1960. The acetolysis method. A revised description. Svensk Botanisk Tidskrift 39:561-564.

ERDTMAN, G. 1965. Pollen and spore morphology/ plant taxonomy. Gymnospermae, Bryophyta (Text). An introduction to palynology III. Almqvist \& Wiksell, Stockholm.

ESTEVES, L.M. \& MELHEM, T.S. 1992. Morfologia de esporos de pteridófitas do cerrado da Reserva Biológica de Moji-Guaçu (SP). Revista Brasileira de Botânica 15:73-83.

FARIAS, H.C. 1984. A família Polytrichaceae no Rio Grande do Sul, Brasil. I Taxonomia. Iheringia (Série Botânica) 32:77-89.

FERRARINI, E., CIAMPOLINI, F., PICHI, R.E.G., SERMOLLI, F.M.L.S. \& MARCHETTI, D. 1986. Iconographia palynologica pteridophytorum Italiae. Webbia 40:1-202.

GONÇALVES-ESTEVES, V. \& MELHEM, T.S. 2000. Palinotaxonomia de espécies brasileiras de Salacia L. (Hippocrateaceae). Revista Brasileira de Botânica 23: 425-440.

HAMPE, E. 1872. Musci frondosi. In: Symbolae ad floram Brasiliae centrales cognoscendam (E. Warming, ed.). Videnskabelige Meddedelelser fra Dansk, Naturhistorisk Forening i Kjøbenhavn ser. 3, 4:53.

HOLMGREN, P.K., HOLMGREN, N.H. \& BAINETT, L.G. 1990. Index herbariorum. Part 1. The herbaria of the world. New York Botanical Garden, New York.

HYVÖNEN, J. 1989. A synopsis of genus Pogonatum (Polytrichaceae, Musci). Acta Botanica Fennica 138:1-87.

HYVÖNEN, J., HEDDERSON, T.A., MERRIL, G.L.S., GIBBINGS, J.G. \& KOSKINEN, S. 1998. On phylogeny of the Polytrichales. The Bryologist 101:489-504.

LUIZI-PONZO, A.P. \& BARTH, O.M. 1998. Spore morphology of some Bruchiaceae species (Bryophyta) from Brazil. Grana 37:222-227. 
LUIZI-PONZO, A.P. \& BARTH, O.M. 1999. Spore morphology of some Dicranaceae species (Bryophyta) from Brazil. Grana 38:42-49.

LUIZI-PONZO, A.P. \& MELHEM, T.S. 2006. Palinotaxonomia de Rhachitheciaceae (Bryophyta) do Brasil. Boletim do Instituto de Botânica 18:91-99.

LUIZI-PONZO, A.P., BARTH, O.M. \& LISBOA, R.C.L. 1997. Estudos palinológicos em briófitas. In Caxiuanã (P.L.B. Lisboa, ed.). Museu Paraense Emílio Goeldi \& MCT/CNPq, Belém, p.305-318.

MENZEL, M. 1985. Die Gattung Pogonatum P. Beauv. (Polytrichales, Musci) in Lateinamerika 1. Taxonomie und geographie von Pogonatum campylocarpum (C. Muell) Mitt. Und P. subflexuosum (Lor.) Broth. Lindbergia 11:134-140.

MCCLYMONT, J.W. \& LARSON, D.A. 1964. An electronmicroscope study of spore wall structure in the Musci. American Journal of Botany 51:195-200.

MOGENSEN, G.S. 1981. The biological significance of morphological characters in Bryophytes. The spore. The Bryologist 84:187-207.

MOGENSEN, G.S. 1983. The spore. In: New manual of bryology (R. M. Schuster, ed.). Nichinan, The Hattori Botanical Laboratory, Myazaki, p.326-341.

MULLEN, D. \& FRYE, T.C. 1947. Middle and South American species of Oligotrichum. The Bryologist 50:67-79.

NYHOLM, E. 1971. Studies in the genus Atrichum P. Beauv. A short survey of the genus and the species. Lindbergia $1: 1-33$.
OLESEN, P. \& MOGENSEN, G.S. 1978. Ultrastructure, histochemistry and notes on germination stages of spores in selected mosses. The Bryologist 81:493-516.

PUNT, W., NILSON, S., BLACKMORE, S. \& LE THOMAS, A. 2007. Glossary of pollen and spore terminology. http://www.bio.uu.nl/ palaeo/glossary/glos-int.htm (acesso em 03/09/2007).

SCHOFIELD, W.B. 1985. Introduction to bryology. MacMillan Publishing, New York.

SMITH, G.L. 1971. Conspectus of the genera of Polytrichaceae. Memoirs of The New York Botanical Garden 21:1-83.

SMITH, G.L. 1974. New developments in the taxonomy of Polytrichaceae: Epiphragm structure and spore morphology as generic characters. Journal of the Hattori Botanical Laboratory 38:143-150.

STEFUREAC, T.I., PLOIAE, G. \& PLOIAE, P. 1979. Surface ultramorphology of the spores of some species of Polytrichaceae. Pollen et Spores 21:279-286.

SYLVESTRE, L.S. 1995. Palinologia das Polypodiaceae sensu lato do planalto de Itatiaia, Rio de Janeiro, Brasil. Arquivos do Jardim Botânico do Rio de Janeiro 33:9-73.

$\mathrm{W}^{3}$ Most (Moss Database Nomeclature) - Missouri Botanical Garden. 2006. http://mobot.mobot.org/W3T/Search/ most.html (acesso em 15/04/2006).

WODEHOUSE, R.P. 1935. Pollen grains. Their structure, identification and signification in science and medicine. McGraw-Hill Book Company, New York.

YANO, O. 1996. A checklist of brazilian bryophytes. Boletim do Instituto de Botânica 10:47-232. 